\title{
Russie : un retour réussi sur la scène agricole mondiale. Des années 1990 à l'embargo
}

Russia: A successful comeback on the world agricultural market. From the $1990 \mathrm{~s}$ to the embargo

\section{Quentin Mathieu et Thierry Pouch}

\section{(2) OpenEdition}

\section{Journals}

Édition électronique

URL : http://journals.openedition.org/economierurale/6038

DOI : 10.4000/economierurale.6038

ISSN : 2105-2581

Éditeur

Société Française d'Économie Rurale (SFER)

Édition imprimée

Date de publication : 30 septembre 2018

Pagination : 103-118

ISSN : 0013-0559

Référence électronique

Quentin Mathieu et Thierry Pouch, «Russie : un retour réussi sur la scène agricole mondiale. Des années 1990 à l'embargo », Économie rurale [En ligne], 365 | Juillet-septembre 2018, mis en ligne le 30 septembre 2020, consulté le 05 janvier 2020. URL : http://journals.openedition.org/economierurale/ 6038 ; DOI : 10.4000/economierurale.6038

(c) Tous droits réservés 


\title{
Russie : un retour réussi sur la scène agricole mondiale Des années 1990 à l'embargo
}

\author{
Quentin MATHIEU • Service études, références et prospective Assemblée Permanente \\ des Chambres d'Agriculture-Paris \\ quentin.mathieu@apca.chambagri.fr \\ Thierry POUCH • Université de Reims Champagne-Ardenne Laboratoire REGARDS. Service \\ études, références et prospective Assemblée Permanente des Chambres d'Agriculture-Paris \\ thierry.pouch@apca.chambagri.fr
}

L'instauration par Moscou d'un embargo commercial à l'endroit des produits agricoles et alimentaires en provenance de l'Union européenne, des États-Unis et de quelques autres pays comme la Norvège ou l'Australie a eu des répercussions importantes. Ces répercussions n'ont toutefois été mesurées que du seul côté des exportateurs. Le préjudice économique subi, notamment par des producteurs français de viandes, de fruits et légumes et de produits laitiers, a été rapidement mis en avant. Sans sous-estimer l'importance des pertes occasionnées par la fermeture du marché russe, il semblait intéressant de voir quelles ont pu être les conséquences de l'embargo côté russe. L'article montre que cette politique commerciale n'a fait qu'amplifier une tendance à l'œuvre depuis le début des années 2000, à savoir le redressement de l'agriculture russe. La Russie est ainsi (re)devenue l'un des grands acteurs agricoles mondiaux.

MOTS-CLÉS : relations internationales, politique commerciale, production et échanges agricoles et alimentaires

\section{Russia: A successful comeback on the world agricultural market. From the 1990 s to the embargo}

Russia's ban on imported food and agricultural products from the European Union, the United States, as well as from other countries such as Norway and Australia, has had significant impacts. However, these impacts have only been assessed from the exporters' point of view, with, for example, people being quick to stress the economic damage done to French meat, dairy, fruit, and vegetable producers. Without playing down the importance of the losses related to the closure of the Russian market, it seemed relevant to further investigate the impacts that Russia's import ban has had on Russia itself. This article shows that this trade policy has reinforced a long-term trend observed since the 2000s: the successful recovery of Russian agriculture. Thus, Russia remains one of the world's major players in agriculture. (JEL: Q17, Q18, F51, F52).

KEYWORDS: international relationships, trade policy, agricultural and food production, agricultural and food trade

$\mathrm{D}$ écrété à la suite des sanctions financières infligées par les pays occidentaux dans le contexte de la crise ukrainienne, l'instauration par Moscou d'un embargo commercial durant l'été 2014 à l'encontre des produits agricoles et alimentaires en provenance des États-Unis, de l'Union européenne, du Canada, de Norvège et d'Australie, a très vite suscité deux types d'interrogations. Le premier a trait aux conséquences commerciales de cet embargo sur les pays exportateurs 
de biens agricoles et alimentaires vers la Russie. Dans le cas spécifique de l'Union européenne, la fermeture du marché russe a intensifié la concurrence intra-communautaire puisque les principaux exportateurs ont cherché des débouchés de substitution à leurs produits sur le marché intérieur européen ${ }^{1}$. Le second type de questionnement a concerné les modalités de l'approvisionnement alimentaire de la Russie. Des sources d'approvisionnements alternatives ont-elles été recherchées et trouvées par Moscou auprès de nouveaux fournisseurs ? Au passage, le rationnement de l'offre de produits alimentaires sur le marché russe suggère de mesurer l'inflation sur les prix alimentaires.

Alors que l'embargo est actif depuis plus de trois ans, que de nombreux acteurs économiques russes encouragent le gouvernement à le proroger, un troisième type de questionnement, complémentaire des deux précédents, mérite d'être mis au jour. Il s'agit de l'impact de l'embargo sur la production intérieure agricole et de produits transformés. Comment cette production a-t-elle évolué depuis l'entrée en application de cet embargo russe ? Puisque le marché russe était moins, voire plus du tout, approvisionné par le truchement des importations, l'effet incitatif sur la production intérieure a-t-il été suffisamment puissant pour restaurer la capacité d'auto-approvisionement du pays ? Autrement dit, la Russie a-t-elle pratiqué ce que les économistes du développement nommaient autrefois un processus de " substitution d'importations », au profit d'une " croissance autocentrée » de la production agricole, qui, conformément à sa définition, trouve sur le marché intérieur les débouchés suffisants à sa production?

1. https://www.lesechos.fr/idees-debats/cercle/ cercle-173171-lembargo-alimentaire-a-t-il-profite-a-la-russie-2110012.php, article consulté le 9 janvier 2018.
Cet article se propose d'apporter des éléments de réponse à cet ensemble de questions. Il s'inscrit dans une réflexion plus vaste relative au retour de la Russie sur l'échiquier agricole international, en rupture avec l'évolution empruntée après le passage à l'économie de marché. Lorsque l'on se penche sur la dynamique de la production agricole en Russie, on constate que la trajectoire du redressement productif était engagée bien avant l'embargo. Celui-ci n'a fait en réalité qu'accentuer une évolution déjà à l'œuvre depuis le début de la décennie 2000. Il est donc suggéré de mettre en perspective historique la croissance de la production agricole russe. C'est pourquoi la première partie de l'article traitera de manière synthétique de l'effondrement de la production agricole russe durant les années 1990, à la suite de la transition économique. Elle entend aussi montrer que des facteurs de redressement sont à l'œuvre dès le début des années 2000, facteurs ayant ouvert pour la Russie la voie à un positionnement d'exportateur net, notamment en céréales.

La seconde partie examine la dynamique des échanges commerciaux agroalimentaires de la Russie. Ce point a son importance non seulement pour montrer en quoi la Russie reste globalement dépendante de l'extérieur pour ses approvisionnements alimentaires, mais aussi dans la mesure où l'analyse de ces flux commerciaux depuis la fin de l'année 2014 permet de voir en quoi l'embargo a occasionné une réduction du déficit russe, tout en modifiant la hiérarchie des nations exportatrices de céréales, et en quoi ils se superposent aux intérêts géopolitiques de Moscou. Il est par conséquent suggéré de faire un bref détour par la théorie des sanctions internationales, qui, en tant que branche de l'économie internationale, évalue l'efficacité de mesures définies et appliquées par les pouvoirs publics, comme l'embargo, afin de comprendre 
l'ambition de la Russie il y a bientôt quatre ans. Il s'inscrit en effet dans un contexte nouveau, où s'entremêlent les dimensions géopolitiques et géoéconomiques, et où des puissances émergentes - c'est le cas de la Russie - entendent reconfigurer la hiérarchie des nations en matière de production et d'échanges de biens agricoles et alimentaires.

\section{Héritage soviétique et développement agricole}

\section{Une structuration des exploitations agricoles russes en partie basée sur le legs soviétique}

À l'image de son économie, l'agriculture russe a connu une transformation radicale de sa structure en l'espace de trente ans d'histoire. La chute de l'Union soviétique au début des années 1990 a laissé un immense chantier dans l'espace rural, où l'échec de la planification n'en a pas moins gardé un système agricole marqué par la collectivisation des terres, illustrée par les kolkhozes et les sovkhozes. Cet héritage institutionnel est essentiel pour comprendre la structuration des fermes agricoles russes d'aujourd'hui.

La première réforme agraire de 1991, initiée par Boris Elstine, consista à promouvoir la propriété privée en redistribuant près de $62 \%$ des terres agricoles sur la période 1991-1993, soit plus de 115 millions d'hectares (Rosreestr, 2012) aux collectifs d'anciens employés des kolkhozes sous la forme de coupons faisant office de titres de propriété. Mais cette restructuration du foncier, s'inscrivant dans le processus de libéralisation économique enclenché par le consensus de Washington, amena une profonde déstructuration de l'agriculture russe. Cette politique se révéla inefficace pour stimuler la production agricole, celle-ci ayant drastiquement chuté dès le début de la transition vers l'économie de marché de la Russie. L'échec de cette réforme provient de plusieurs facteurs, traduisant une mesure politique précipitée et désorganisée dans le contexte de libéralisation de l'économie russe (Yefimov, 2001 ; Néfédova et Eckert, 2003 ; Kirtchik, 2009).

En effet, le partage des terres qui était proposé aux détenteurs de titres de propriété ne prévoyait aucun découpage cadastral de la propriété collective, ce qui revenait à fractionner la propriété à parts quasiment égales entre les détenteurs de coupons, mais sans indiquer la localisation des parcelles. La réforme ne permettait pas non plus de détacher le terrain de l'ancienne propriété collective. Par ailleurs, la plupart des détenteurs de titre de propriété de terre agricole ne souhaitaient pas devenir des fermiers indépendants et être confrontés aux risques de marché, préférant alors rester comme employés sur l'exploitation. De plus, la taille de ces terres se montrait bien trop importante pour intéresser les fermiers les plus modestes, alors que la vétusté et le manque de matériel agricole pénalisaient l'exploitation de ces terres. Enfin, la forte croissance de la production agricole des pays européens voisins représentait aussi une opportunité pour la Fédération de Russie de s'approvisionner en denrées alimentaires à coûts raisonnables évinçant ainsi les exploitations russes incapables de rivaliser en termes de rentabilité et de compétitivité. Une orientation appuyée par la volonté des autorités russes de réduire les soutiens au secteur agricole durant la vague de libéralisation impulsée par le consensus de Washington (Barsukova, 2016a). Conséquence, la production de grains et de viandes chuta de respectivement $43 \%$ et de $55 \%$ entre 1990 et l'année $2000^{2}$.

2. Source : calculs des auteurs, d'après les données de l'USDA Foreign Agricultural Service. 
Une nouvelle législation sur la vente des terres fut promulguée entre 2001 et en 2002 par l'adoption d'un nouveau Code foncier et d'une loi sur les terres agricoles, permettant de mieux encadrer les transactions, notamment grâce à la redéfinition des limites cadastrales des parcelles et à l'officialisation de l'acte de propriété (Lezean, 2011 ; Rylko, Khotko, Svetlana, Yunosheva et Glazunova, 2015). Même si la séparation des terrains vis-à-vis de l'ancienne propriété collective demeurait difficilement applicable, cette seconde réforme s'inscrit comme l'acte inaugural du redressement de la production agricole russe, qui connaîtra par la suite une croissance ininterrompue. Il faut aussi ajouter que cette réforme fut élaborée peu de temps après que l'économie russe eut subi les répercussions de la crise financière de 1998 dès l'année suivante, entraînant une forte dévaluation du rouble, une fuite de capitaux et un retrait des importations de produits alimentaires.

Cette crise eut le mérite de rendre à nouveau le secteur agricole attractif, du fait de l'envolée du coût des importations et de la perte de valeur du rouble. Les puissants oligarques russes, soutenus par les pouvoirs politiques locaux, ont profité de cette opportunité pour diversifier leur portefeuille d'activités en rachetant d'anciens kolkhozes et sovkhozes (Grouiez, 2012a). De son côté, le secteur de l'industrie agroalimentaire et de la grande distribution russe, qui s'approvisionnait en majorité à l'étranger, a constaté que sa dépendance aux importations le rendait considérablement fragile en cas de crise. Or le démantèlement incomplet des anciennes fermes collectives a contribué à préserver de façon sous-jacente le fonctionnement hiérarchique de celles-ci sous l'ancien régime soviétique, à savoir le contrôle de l'exploitation par le chaînon aval de la filière. Ainsi, avec l'apport des capitaux par des investisseurs cherchant à diversifier leurs activités ou bien à sécuriser leurs approvisionnements, le maintien de ces exploitations de grandes tailles représentait un terreau extrêmement favorable pour la création de chaîne de valeur verticalement intégrée, comme avec les agro-holdings entre autres (Cordonnier et Hervé, 2003 ; Grouiez, 2012a). Cette nouvelle orientation du modèle agricole fut définitivement actée lors du premier mandat de Vladimir Poutine, au cours duquel l'agriculture fut déclarée « priorité nationale » en 2005 avant d'être l'objet d'un premier programme d'État de 2008 à 2012.

Ce plan prévoyait un programme d'investissement de 26 milliards de dollars afin de moderniser et de renforcer la production agricole locale, mais sans remettre en cause l'autosuffisance alimentaire et la dépendance du pays aux importations agroalimentaires. Par conséquent, le développement des agro-holdings russes ne fut pas suivi d'une réduction des importations agroalimentaires du pays. Celles-ci s'intensifièrent jusqu'à atteindre 43 milliards de dollars en 2013, soit un an avant la mise en place de l'embargo commercial à la suite du conflit en Crimée et des sanctions internationales.

\section{Une politique agricole nationale longue à se dessiner}

Cette disposition de l'agriculture russe, couplant le développement de la production locale et l'expansion des importations, peut sembler contradictoire mais réside dans une approche duale de l'économie russe et de son agriculture plus particulièrement. La promotion du libéralisme par les autorités politiques s'est heurtée aux représentants du monde agricole russe, adeptes d'une conception davantage planifiée de ce secteur. Le développement du libéralisme après la chute du Mur de Berlin fut rapidement confronté aux réalités agraires, où la croyance envers les 
vertus du marché n'a pas provoqué d'élan productif dans les campagnes. Malgré cette chute évidente de la production agricole et les inquiétudes sur la capacité du pays à satisfaire les besoins alimentaires de sa population, les autorités agraires russes n'ont pas réussi à peser dans les débats publics. Et ce malgré plusieurs injonctions adressées aux gouvernements successifs remettant en cause la dépendance grandissante du pays aux importations de biens alimentaires $^{3}$. En 2005, le taux de dépendance aux importations alimentaires avait atteint plus de $30 \%$, sans pour autant que la Russie ne soit encore membre de l'Organisation mondiale du commerce (OMC).

En effet, malgré la dizaine de rounds de négociations d'adhésion de la Russie, l'opposition de la communauté agraire russe aux trop fortes concessions demandées par les pays membres de l'OMC allongea considérablement le processus d'adhésion. Les principaux points de blocages résidaient dans la réduction des mesures globales de soutien, alors que le secteur agricole russe, en pleine décroissance à la fin des années 1990, avait vu ses subventions se réduire drastiquement lors de la crise financière de 1998 (voir graphique 1). D'autre part, le régime commercial se trouvait déjà extrêmement libéralisé pour pallier les insuffisances de la production nationale, ce qui désavantageait les complexes agro-industriels russes encore convalescents. Ces derniers ne pouvaient se montrer aussi compétitifs que les industries agroalimentaires des pays développés, alors que ces dernières

3. Exemple avec la proposition d'un arrêté intitulé : « De la sauvegarde de la sécurité alimentaire de la Fédération de Russie », adopté par le Conseil de la Fédération et de la chambre haute du Parlement le 5 juillet 1995. Le texte de loi « De la sécurité alimentaire » qui en découlait fut rejeté par le président russe en 1998, sans examen (Kirtchik, 2009). bénéficiaient d'un niveau de soutien et de protection bien plus avantageux.

Ce n'est qu'au début des années 2000 que le secteur agricole russe fut bénéficiaire d'un réel décollage de la politique de développement rural instauré par Moscou, avec des déclarations d'intentions en matière d'objectifs de sécurité alimentaire qui seraient atteints grâce à une politique favorable au développement des grandes exploitations. Ce dernier point s'est traduit par une allocation des ressources budgétaires envers les exploitations avec une situation financière viable, et ceci afin de faire émerger des réseaux d'exploitations intégrées dans des structures du type agro-industriel (Lezean, 2011). Cette orientation fut préjudiciable aux petites exploitations, et dont la contribution à la production agricole diminua fortement, en particulier pour les productions animales (Lezean, 2011 ; Barsukova et Zvyagintsev, 2015 ; Barsukova 2016a, 2016b). L'émergence d'une telle technostructure fut possible, d'une part, grâce au soutien financier de la Fédération à ses localités rurales, jouant désormais le rôle de centres de gestion ainsi que de services, et ont pour mission de favoriser la création d'exploitations et de toutes formes de coopération auxquelles elles sont liées (coopératives, organismes de micro-crédit). D'autre part, il faut également prendre en compte l'impact de l'arrivée de Vladimir Poutine au pouvoir sur les institutions russes. En neutralisant le pouvoir dont disposaient les gouverneurs régionaux en termes d'application des lois foncières, plus particulièrement grâce à l'adoption d'un nouveau cadre réglementaire favorable aux rachats des exploitations par des opérateurs privés, et du fait des liens étroits entretenus par la nouvelle administration avec les milieux industriels et financiers, la formation de ces grands complexes agro-industriels fut 


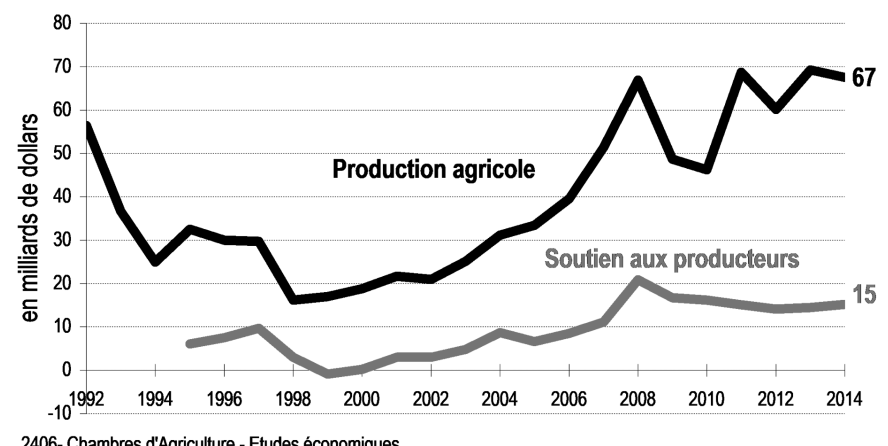

Source : $F A O$ et $O C D E$.

Tableau 1. Principales productions agricoles par type d'exploitation (en \% du total de la production)

\begin{tabular}{|c|c|c|c|c|c|c|}
\hline \multirow[t]{2}{*}{ Produits } & \multicolumn{2}{|c|}{ Exploitations agricoles } & \multicolumn{2}{|c|}{$\begin{array}{l}\text { Exploitations } \\
\text { paysannes }\end{array}$} & \multicolumn{2}{|c|}{$\begin{array}{l}\text { Exploitations } \\
\text { auxiliaires }\end{array}$} \\
\hline & 1992 & 2005 & 1992 & 2005 & 1992 & 2005 \\
\hline Céréales & 96,9 & 80,2 & 3,1 & 18,3 & - & 1,5 \\
\hline Betterave sucrière & 98,0 & 87,8 & 2,0 & 10,4 & - & 1,8 \\
\hline Graines de tournesol & 95,3 & 72,5 & 4,7 & 26,6 & - & 0,9 \\
\hline Pommes de terre & 13,7 & 6,3 & 0,4 & 2,1 & 85,9 & 91,6 \\
\hline Légumes & 46,1 & 14,0 & 0,9 & 5,7 & 53,0 & 80,3 \\
\hline Viande & 63,4 & 45,7 & 0,7 & 2,5 & 35,9 & 51,8 \\
\hline Lait & 61,7 & 45,2 & 0,6 & 3,0 & 37,7 & 51,8 \\
\hline CFufs & 59,0 & 74,1 & 0,1 & 0,7 & 40,9 & 25,2 \\
\hline
\end{tabular}

favorisée avec cette incursion du secteur privé dans la sphère publique.

Ainsi, l'adoption de la loi de 2006 sur le développement de l'agriculture apporta une orientation claire à la politique agricole russe, se fondant sur deux grands principes : l'approvisionnement de la population en produits agricoles russes et le développement durable des territoires ruraux. Le relèvement significatif des aides et de la production agricole traduit bien cette nouvelle inflexion de la politique russe, avec un niveau moyen des mesures de soutien aux producteurs qui est passé de 4,1 milliards de dollars sur la période
1998-2006 à 15,5 milliards de dollars sur la période 2007-2014 (graphique 1).

La mise en place d'une réelle politique agricole nationale, couplée à des structures d'exploitations héritées des sovkhozes et des kolkhozes, a ainsi permis de confirmer cette dynamique dans les territoires ruraux russes, de même que de valider le processus de concentration des exploitations russes. Ainsi, en 2008, près de $20 \%$ des exploitations agricoles détenaient $60 \%$ de la surface agricole du pays, ces exploitations ayant une taille comprise entre 6000 et plus de 10000 ha (graphique 2). Cependant, ce processus cache également de profondes disparités 
selon les types de production qui, pour certaines d'entre elles, sont restées la propriété des très petites exploitations. En 2005, à titre d'exemple, $90 \%$ de la production nationale de pommes de terre était assurée par ce type d'exploitation, $80 \%$ pour les légumes (tableau 1).

Il convient aussi d'évoquer les nouvelles relations que nouent ces structures agro-industrielles avec les localités, dans le sens où elles peuvent être amenées à fournir des biens et des services collectifs en échange de facilités administratives et/ou financières. Par exemple, l'administration publique régionale fournit des subventions aux agro-holdings, qui prennent ensuite en charge le financement des biens et de services collectifs (route, réseau de gaz et d'électricité, logement pour les employés) dans les localités où se situent leurs installations et/ou les agriculteurs avec qui elles contractent (Grouiez, 2011).

Il est également important de signaler l'impact de la réglementation sur les importations de viandes mise en place entre 2003-2006, ainsi que la création d'un plan d'aide à l'investissement fondé sur un système de subventions sur les taux d'intérêt. Par exemple, le système de quotas sur les importations de volaille et de contingents tarifaires sur le bœuf et le porc ont fonctionné comme des mécanismes d'incitation à l'investissement pour les agro-holdings et les industries agroalimentaires. Ceci est particulièrement vrai pour les productions à cycle court et à retour sur investissement rapide que sont la volaille et le porc, celles-ci ayant été respectivement multipliées par 2 et par 4 entre 2004 et 2015 (graphique 3). La production de viande bovine reste sur une tendance légèrement en baisse du fait d'un manque d'intérêt des investisseurs à placer des capitaux dans une production à cycle long, et donc moins rentable à court terme. Mais globalement, l'effet de ces mécanismes fut bénéfique puisque, d'une part, les importations de viandes ont commencé à diminuer dès 2007 et que, d'autre part, le taux d'autosuffisance en viande est passé de $65 \%$ en 2004 à plus de $88 \%$ en 2015 (graphique 4).

Toutefois, ce dernier élément est quelque peu tronqué par la forte inflation alimentaire qu'a connue le pays après l'embargo de 2014 et son impact sur les comportements de consommation des ménages, avec, à la fois, un effet de revenu et un effet de substitution (moins de produits alimentaires consommés, substitution de

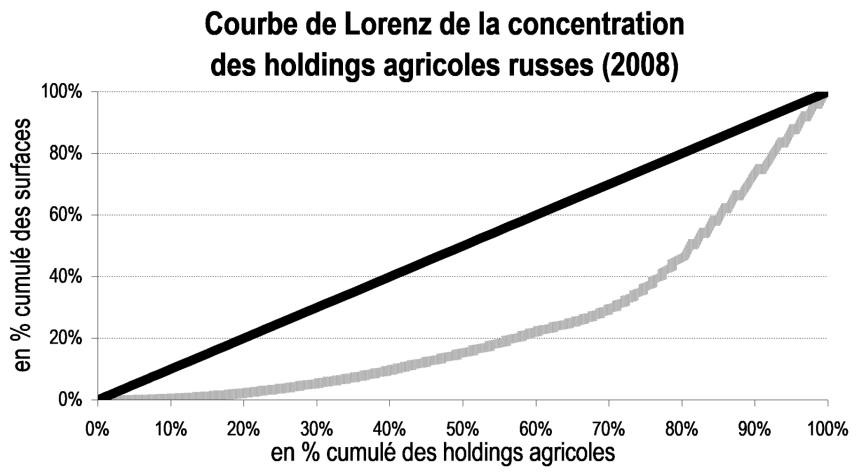

2406- Chambres d'Agriculture - Etudes économiques

Source : Rosstat, d'après IKAR. 

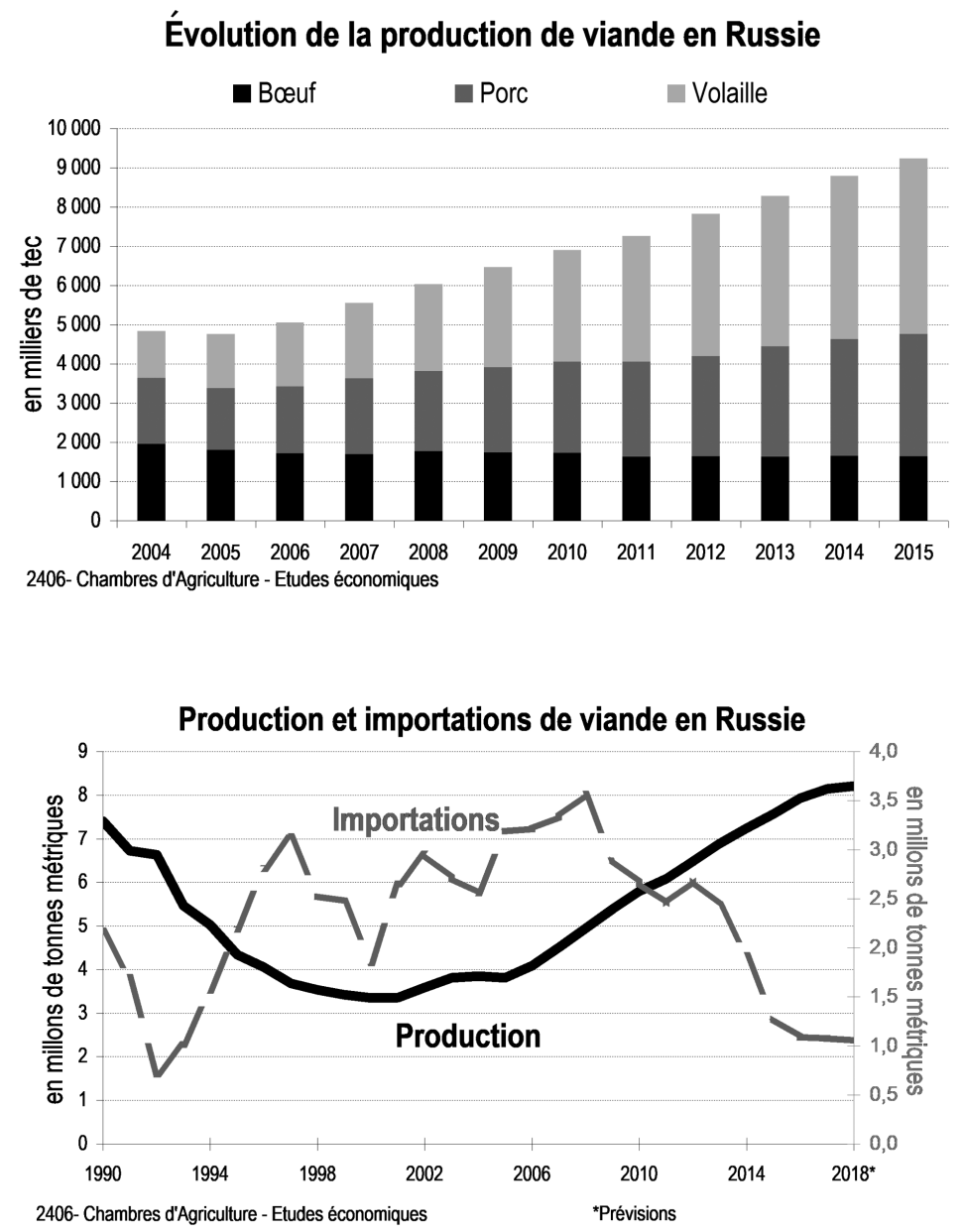

Source : Rosstat et USDA.

la consommation de viande bovine à prix élevé au profit de protéines animales meilleures marchés comme le poulet ou les œufs). Les conséquences sur la consommation alimentaire des ménages russes ont été particulièrement fortes, les prix des produits agroalimentaires ayant augmenté en moyenne de plus de $26 \%$ entre août 2014 et août 2016 (avec des hausses plus marquées pour les produits de la pêche ou les fruits et légumes), ce qui a entraîné une baisse des achats sur les produits alimentaires les plus coûteux dans le panier de biens des ménages : $-5 \%$ par habitant pour les fruits, $-4 \%$ pour les produits laitiers et $-3 \%$ pour les viandes entre 2013 et $2015^{4}$.

4. https://financialobserver.eu/cse-and-cis/ russia/the-embargo-has-transformed-the-russian-food-market/, article consulté le 18 janvier 2018. 
L'embargo a également eu un impact qualitatif sur cette consommation alimentaire, le service fédéral de surveillance vétérinaire et phytosanitaire (Rosselkhoznador) ayant mis en évidence une recrudescence de produits alimentaires falsifiés dans les circuits de distribution. Ceci concernerait un quart de la production alimentaire totale et jusqu'à $78 \%$ des fromages, des compagnies corrompues y incorporant des huiles végétales en substitution du lait.

\section{Une balance agroalimentaire déficitaire, et les conséquences de l'embargo commercial}

\section{Production agricole relancée, mais un déficit de la balance commerciale agroalimentaire}

Ce désintérêt pour la production de viande bovine est étroitement lié au déficit de croissance du secteur laitier russe. La production du lait en Russie stagne depuis le début des années 2000 (entre 30 et 32 millions de tonnes sur les quinze dernières années). Cette situation est la conséquence d'un sous-investissement chronique dans le secteur laitier du fait des délais particulièrement longs pour récupérer les capitaux investis, contrairement à des productions sur cycle court. Ainsi, la défaillance dans le processus de modernisation du secteur a maintenu la productivité par vache à un niveau deux fois et demie inférieur aux autres grands bassins mondiaux de production, et en sachant que seulement la moitié de la production est collectée puis transformée ${ }^{5}$. Contrairement au secteur de la viande, le pays a dû continuer ses importations de produits laitiers, les capacités de transformation y sont encore insuffisamment nombreuses et performantes pour satisfaire la demande

5. https://www.lecourrierderussie.com/econo$\mathrm{mie} / 2017 / 03 /$ nouveaux-investisseurs-secteur-agro-industriel/, article consulté le 15 janvier 2018. intérieure. Ainsi, entre 20 et $25 \%$ de la consommation laitière russe provient des importations, plus particulièrement sur des produits à forte valeur ajoutée comme les fromages ou le beurre ${ }^{6}$.

L'exemple de la filière laitière est symbolique du manque de modernisation dont souffrent encore les filières agricoles russes, et qui a conduit à la dégradation de la balance commerciale agroalimentaire du pays, malgré une amorce de croissance de sa production agricole. La moitié des semences utilisées est importée de l'étranger ( $95 \%$ en provenance de France), le matériel agricole est vétuste, les capacités de transformation et certaines filières accusent toujours un fort déficit d'investissement et de compétences, comme la culture de fruits et légumes sous serre. Sur ce dernier point, le cas est particulièrement problématique puisque le pays consomme de 1,7 à 1,8 million de tonnes de fruits et légumes par an, alors que la production atteint péniblement les 850000 tonnes en moyenne ces dernières années.

C'est dans ce cadre que fut lancé le second plan d'investissement pour l'agriculture sur la période 2013-2020, un an avant l'embargo commercial décrété par Moscou, en réponse aux sanctions financières décrétées à la suite du conflit en Crimée. L'enveloppe budgétaire a été doublée par rapport au précédent plan de 2008-2012 (plus de 52 milliards de dollars), une part des aides est désormais réorientée vers les secteurs en retard de croissance et une nouvelle orientation a été ajoutée dans cette politique : la substitution aux importations pour parvenir à l'autosuffisance alimentaire.

6. En 2013, et d'après les calculs effectués sur les données Rosstat et de l'USDA, nous constatons que la part des importations russes dans la consommation domestique atteint $40 \%$ pour les fromages et le beurre, et $30 \%$ pour le lait cru. Ces taux se situent désormais entre 25 et $30 \%$ après embargo. 
Ce dernier point est particulièrement important puisque le déclenchement de ce nouveau plan intervient seulement un an après l'adhésion de la Russie à l'OMC, alors que le pays a fait d'importantes concessions en matière de soutiens financiers et de protectionnisme pour son agriculture et pour intégrer l'OMC. À défaut de pouvoir davantage accroître les droits de douanes pour limiter l'entrée de produits étrangers plus compétitifs sur son marché intérieur, le pays a intensivement eu recours aux barrières non tarifaires pour contenir des importations alimentaires destinées à s'accroître avec l'entrée dans l'OMC. Le but étant ici, sur la base de la théorie de la protection des industries naissantes de F. List $(1841)^{7}$, de continuer à protéger un secteur agricole encore considéré en transition et moins compétitif que celui des pays développés ou du groupe des Cairns (Brésil, Argentine entre autres).

Ce contrepoids au cadre de l'OMC fut aussi possible grâce à la création d'une Union douanière avec la Biélorussie et le Kazakhstan dès le début de l'année 2010. En remarque, la création de cette union était le fruit d'un long processus de négociation entre les pays participants, puisque les discussions furent entamées dès les années 1990, suivies de la signature de premiers documents officiels en 2006. Cette Union représenta une opportunité pour les opérateurs russes, afin de l'utiliser comme un espace d'écoulement des productions destinées à l'exportation (les grains) mais aussi, dans le cas de la Biélorussie, tel un circuit d'approvisionnement en produits où le secteur agricole russe est déficitaire, comme les produits laitiers (Grouiez, 2012b).

7. List F. (1857). Système national d'économie politique. Trad. de l'allemand par Heni Richelot. Paris, Capelle, $2^{\mathrm{e}}$ éd.

\section{L'embargo a contribué à consolider le redressement agricole de la Russie}

Dans de telles dispositions, la survenance de l'embargo russe a provoqué, dans un premier temps, une forte réduction des importations en provenance de l'Union européenne. En 2013, l'Union européenne exportait pour 16 milliards de dollars de produits agroalimentaires en Russie, ce qui représentait $37 \%$ des importations totales de la Russie en produits agroalimentaires. Ce montant est passé à 6 milliards de dollars en 2015 et l'Union européenne ne pèse plus que pour $24 \%$ des importations agroalimentaires de la Fédération ${ }^{8}$. Ce gel des échanges avec les 28 États membres de l'UE a eu pour conséquence de rediriger une partie de ces flux d'importations vers des pays partenaires comme la Biélorussie ou le Brésil, mais aussi vers de nouveaux pays fournisseurs comme la Turquie, le Paraguay ou l'Argentine'. Ces flux d'importations se caractérisent entre autres par une spécialisation de chaque pays fournisseur dans l'approvisionnement de produits agricoles ciblés : les fruits et légumes pour la Turquie, les viandes pour les pays d'Amérique du Sud.

Par ailleurs, la diminution des importations de l'Union européenne dans le commerce russe n'a pas été totalement compensée par cette réorientation des flux d'importations alors que les exportations agroalimentaires de la Russie se sont accrues de près de 8 milliards de dollars entre 2010 et 2016, sous l'impulsion notamment des récoltes records de grains. Ainsi, la balance commerciale

8. Calculs des auteurs, d'après les données de la banque de données CHELEM (Comptes harmonisés sur les échanges et l'économie mondiale), nomenclature GTAP.

9. Il faut préciser ici que les opérateurs européens à l'exportation ont contourné, dans un premier temps, les sanctions russes en procédant à des réétiquetages des produits européens à partir de plates-formes situées en Biélorussie. 


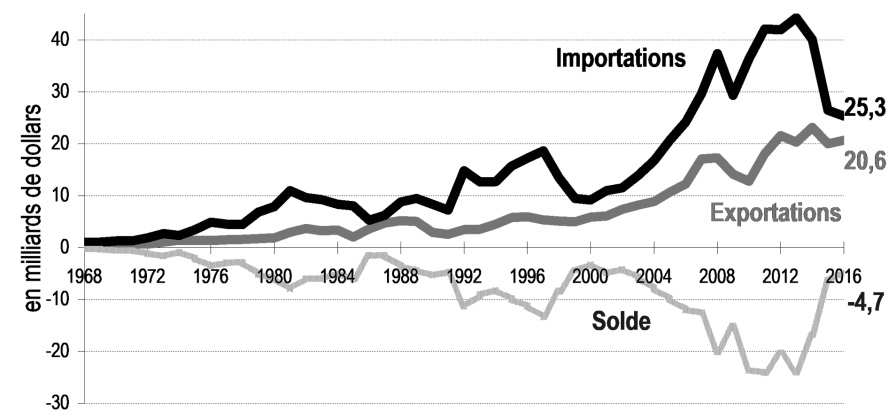

2406- Chambres d'Agriculture - Etudes économiques

Source : CEPII-CHELEM.

Graphique 6. Flux d'IDE entrants en Russie dans le secteur agroalimentaire

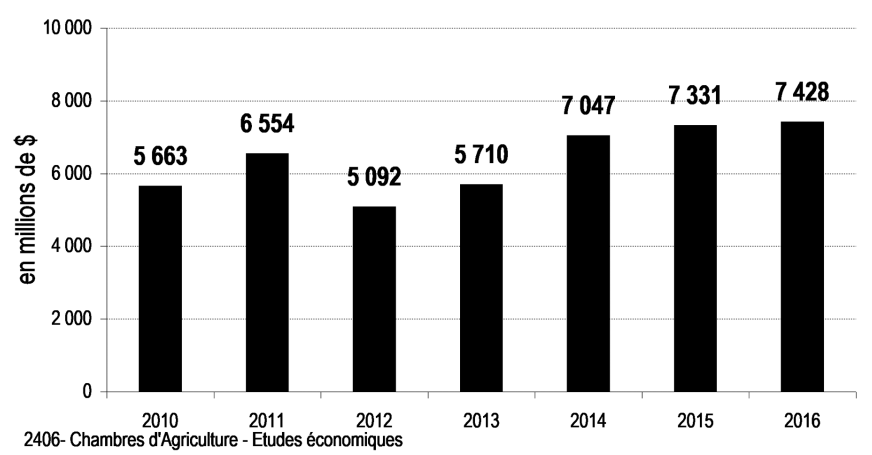

Source : $C B R$.

du pays s'est davantage rééquilibrée, le déficit agroalimentaire russe n'étant plus que de 4,7 milliards de dollars en 2016 contre 24 milliards de dollars en 2013 (graphique 5).

Enfin, les dispositions de l'embargo ont révélé une autre facette de la politique agricole russe qui consiste à développer l'accueil d'investissements agroalimentaires sur le territoire russe. Les flux d'investissements directs étrangers (IDE) dans le secteur agroalimentaire ont continué de progresser, et n'ont jamais été aussi élevés que durant l'embargo (graphique 6). Ce phénomène traduit bien cette stratégie des autorités russes de parvenir à un objectif d'autosuffisance alimentaire, mais tout en continuant à s'intégrer dans la division internationale du travail par la création de partenariats économiques avec des pays qui leur fournissent des intrants, des technologies et des compétences dont ils sont déficitaires.

Une partie de ces IDE a aussi la particularité d'être dirigée vers des filières encore faiblement développées dans le pays. La filière laitière en est le parfait exemple, puisque de nombreux projets de grandes fermes ou de complexes intégrés étaient en cours de construction pour des montants extrêmement élevés. Citons notamment en 2016, un investissement sur 
dix ans du groupe vietnamien TH True Milk pour un montant de 2,4 milliards d'euros dans un complexe d'une capacité d'accueil prévue à 350000 vaches laitières ${ }^{10}$. Le secteur laitier devrait d'ailleurs constituer les prochaines filières réceptrices d'investissements en plus grande quantité, aussi bien étrangers que nationaux, sur la prochaine décennie (de même que la production de légumes sous serre). D'un autre côté, le programme de développement de la production laitière $\mathrm{du}$ gouvernement russe envisage d'augmenter la production laitière de $37 \%$, soit plus de 42 millions de tonnes d'ici 2025, grâce notamment à des taux d'intérêt subventionnés sur les projets d'investissement laitier.

\section{L'agriculture comme levier d'une stratégie géoéconomique}

L'ambition des autorités politiques russes, à terme, est de dégager une production agricole excédentaire destinée à peser dans les relations géoéconomiques des pays partenaires de la Russie. C'est déjà le cas pour le secteur des grandes cultures, pour lequel le pays a retrouvé une balance commerciale positive dès le début des années 2000 avec l'émergence des premières agro-holdings, dans les terres fertiles du type tchernoziom situées dans le sud du pays. La production de grains a ainsi quasiment retrouvé son niveau d'avant la chute du bloc soviétique, à hauteur de 100 millions de tonnes, et les exportations se sont vertigineusement accrues jusqu'à dépasser les 30 millions de tonnes, faisant de la Russie le premier exportateur mondial de blé en 2016 (graphique 7). La dépréciation du rouble - largement déclenchée par l'effondrement du prix du baril de pétrole, qui a

10. Voir pour cela la publication de l'Institut de l'élevage " Marchés mondiaux des produits laitiers - Année 2016. Perspectives $2017 »$. Institut de l'élevage, Dossier Économie, $\mathrm{n}^{\circ} 480$, juin 2017. occasionné une contraction des recettes fiscales perçues par l'État - a par ailleurs bénéficié aux exportations de blé russe, dont la qualité était déjà excellente, en particulier pour la panification ${ }^{11}$.

Par ailleurs, la quantité de terres encore inexploitées, des rendements encore nettement inférieurs aux autres pays producteurs et le besoin de modernisation des infrastructures (silos, chemins de fer) montrent toute la marge d'amélioration dont disposent les filières céréalière et oléagineuse russes. Sans oublier les investissements en cours pour améliorer les capacités de trituration des industries, qui souhaitent occuper une place prépondérante sur le marché des huiles végétales.

La Russie profite en partie de ce potentiel céréalier dans le cadre des relations géoéconomiques qu'elle entretient avec ses pays partenaires. Pour certains pays grands importateurs de céréales, la Russie est même rapidement devenue le partenaire incontournable pour leurs approvisionnements. Ainsi, en 2016, les importations de blé russe représentaient $30 \%$ des importations totales de céréales en Égypte, et $50 \%$ en Turquie. Le pays a aussi répondu présent sur des zones en situation d'urgence alimentaire comme le Yémen, le Nigeria ou la Syrie. Le placement de la Russie dans ces zones apparaît hautement stratégique, et lui donne un certain poids dans les relations qu'elle entretient avec ces États. D'autant plus que, pour certains d'entre eux, leur stabilité politique et sociale peut dépendre de

11. Il faut mentionner ici la taxe à l'exportation mise en place par les autorités russes en 2015 dans le but de freiner la poussée inflationniste des biens alimentaires sur son marché intérieur. Cette taxe fut suspendue dès septembre 2016, celle-ci n'ayant en rien stoppé les ambitions à l'exportation des opérateurs, ni même démontré son efficacité quant à son objectif de réduire l'inflation alimentaire, alors que son coût économique apparaissait bien plus contraignant (Götze, Koester, Glauben et Bulavin, 2015). 
leur capacité à approvisionner leur population en biens alimentaires de base.

\section{Inscrire l'embargo russe dans un contexte de guerre commerciale}

L'exercice du commerce international est souvent considéré comme un vecteur de pacification des relations internationales. Il s'agit d'un message qui structure l'approche de l'échange de marchandises entre les nations. Un tel message remonte au début de la formation de la science économique. De nombreux travaux ont pu élargir cette conviction, en montrant notamment que l'ouverture commerciale entre deux nations réduisait l'escalade militaire (Martin et al., 2008). Il s'ensuit que deux pays qui échangent des marchandises avec une certaine acuité et régularité seraient moins enclins à recourir aux sanctions, celles-ci pouvant être porteuses de pertes économiques (Matelly, Gomez et Carcanague, 2017). L'expérience de l'embargo russe contredit cette posture.

L'UE et la Russie sont deux entités qui entretiennent depuis de longues années un flux commercial intense de produits agricoles et alimentaires. Les sanctions financières européennes ont rapidement occasionné une réponse économique de la Russie, au travers de l'embargo commercial. Ce dernier a eu, selon les travaux de R.C. Porter, un effet immédiat, puisque les flux commerciaux de produits agricoles et alimentaires ont été interrompus au détriment de l'UE, les producteurs ayant perdu un débouché important (graphique 8 sur le cas de la France) (Porter, 1979 ; Lindsay, 1986). L'embargo a pu ainsi contribuer à déstabiliser des filières comme celle de la viande de porc ou des produits laitiers. Ce type de sanction apparaît donc d'autant plus efficace qu'elle cible un domaine sur lequel un pays est spécialisé, comme dans le cas de la France. L'impact est prioritairement d'ordre microéconomique, même s'il se répercute à l'échelle macroéconomique par le truchement des comptes extérieurs (Hufbauer et al., 2007). Les producteurs européens ne sont pas restés passifs et ont cherché des débouchés de substitution, trouvés en particulier sur le marché intracommunautaire, ce qui a eu pour conséquence d'intensifier une concurrence déjà vive entre les pays membres de l'UE et entre les agriculteurs et industriels de la transformation.

L'embargo russe s'inscrit surtout dans une logique géoéconomique ou, si l'on préfère, de guerre commerciale. Alors que l'on aurait pu penser que les sanctions internationales déstabiliseraient l'économie et l'influence géopolitique de la Russie, le pays a au contraire su renforcer sa position sur l'échiquier d'un monde de plus en plus multipolaire. Désormais plus faiblement exposée à un risque de dépendance alimentaire vis-à-vis de ses importations de produits agricoles, les sanctions internationales et la réaction des autorités russes ont été bénéfiques au développement de son secteur agricole (Mattely, Gomez et Carcannague, 2017). L'efficacité de ces sanctions est d'ailleurs clairement mise en doute, certaines filières européennes comme le lait et le porc y ayant perdu un débouché rémunérateur, alors que l'économie russe n'a subi qu'un choc, en partie transitoire, avec une forte inflation alimentaire sur un $a^{12}$. De plus, la réorientation des échanges russes au profit de certains pays tiers (Brésil, Kazakhstan, Turquie, Uruguay, Israël...) a permis

12. Néanmoins, comme il a été dit précédemment, ce choc a pu avoir un impact de plus long terme en influençant les comportements alimentaires des ménages russes, ces derniers ayant pu substituer leur consommation de viandes au profit de protéines moins onéreuses (viande de volaille, œufs et légumes). L'inflation alimentaire avait atteint jusqu'à $26 \%$ en février 2015 , et elle n'était plus que de $5,8 \%$ en moyenne en 2016 , et de 3,5\% sur les deux premiers trimestres de l'année 2017 (données : Trading Economics). 


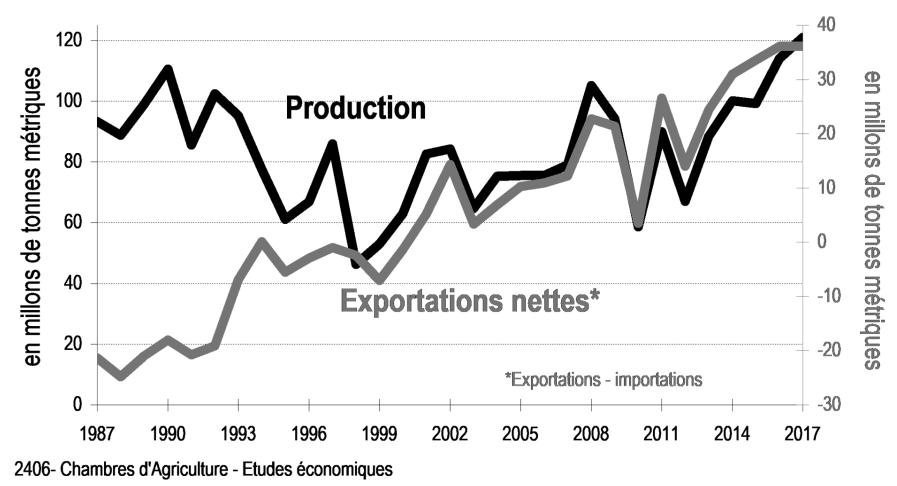

Source : USDA.

au pays de se délester de sa dépendance alimentaire envers l'UE.

Partant de ce qui vient d'être dit, l'analyse de l'embargo russe suggère de se pencher sur la vision que Moscou peut avoir de la sécurité alimentaire, notion revisitée depuis le milieu de la décennie 1990, sous l'impulsion de Via Campesina. Nous savons que la Russie a été confrontée à des formes d'insécurité alimentaire après la dislocation de l'URSS et l'effondrement des productions, incitant les autorités à en faire un objet politique dès le début des années 2000, et surtout lors du gouvernement Medvedev de 2010 (Dufy et Barsukova, 2017). La sécurité alimentaire est depuis perçue en Russie comme un des maillons de la politique de sécurité nationale définie sur l'horizon 2020. L'embargo adopté en 2014 en réaction aux sanctions infligées par l'UE s'inscrit pleinement dans une perception de l'économie répondant à la promotion du nationalisme. La stratégie de Moscou fait écho à l'idée de plus en plus répandue que la sécurité alimentaire ne doit plus forcément passer par l'échange international de produits agricoles et alimentaires (Dufy, 2015).

Nous pourrions donc supposer qu'une levée de l'embargo ne relancerait pas forcément les échanges entre l'UE et la Russie, cette dernière ayant trouvé de nouveaux canaux d'approvisionnements tant en faisant jouer la concurrence internationale que par le redressement de sa production agricole nationale. L'embargo se révèle être un levier supplémentaire de consolidation du regain de croissance de la production agricole russe et de l'élévation de son rang dans les échanges internationaux. Le pays compte du coup asseoir sa position de fournisseur de produits agroalimentaires dans une zone couvrant le Moyen-Orient jusqu'aux grands pays asiatiques. Les filières russes de viande blanche ambitionnent de devenir exportatrices nettes d'ici 2018 pour profiter de ces marchés émergents en très forte demande de produits carnés (la volaille pour l'Orient, la viande porcine pour les pays asiatiques).

Les sanctions financières de l'UE envers la Russie ont donc failli à faire changer le comportement de la Russie en matière de relations internationales. L'effet dissuasif a été très limité. La Russie a en effet pris appui sur ces sanctions pour imposer un embargo qui, non seulement a ouvert la voie à une diversification des approvisionnements alimentaires, mais surtout a renforcé une politique 


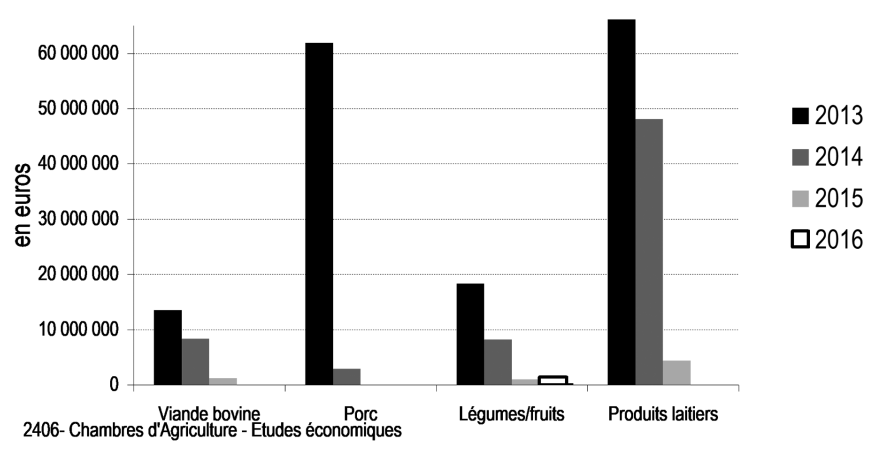

Source : Eurostat.

stratégique engagée au début de la décennie 2000 et visant à faire du secteur agricole un levier du redressement international de la Russie. Avec l'agriculture, nous assistons bel et bien au retour de la Russie, retour qui s'est construit sur une politique macroéconomique, y compris agricole, en opposition franche au credo libéral adopté antérieurement, c'est-à-dire au moment de l'effondrement de l'URSS (Sapir, 2008). De quoi inciter, une fois de plus, à reconsidérer la théorie de l'économie internationale.

\section{RÉFÉRENCES BIBLIOGRAPHIQUES}

Cordonnier C. (2003). Russie. Droit foncier et stratégies agricoles. Le Courrier des pays de l'Est, n 1034/4, pp. 4-14.

Cordonnier C., Hervé J.-J. (2004). Agriculture russe. Les paradoxes du renouveau. Déméter 2004, Économie et Stratégies agricoles, pp. 7-66.

Barsukova S. (2016a) Dilemma fermerj agroholdingi $\mathrm{v}$ contexte importozameshhenija (The Dilemma of the Farmers vs. Agricultural Holdings in the Context of Import Substitution). Obshesvennje Nauki $i$ Sovremennost' (Social Sciences and Contemporaneity), ${ }^{\circ}$ 5, pp. 63-74.

Barsukova S. (2016b) Molochnye reki Rosii (The milk rivers of Russia). ECO 6, $n^{\circ} 504$, pp. 5-17.

Barsukova S., Zvyagintsev V. (2015). Zemel'naja reforma v Rossii v 1990-2000-e gody, ili kak v hode vedomstvennyh reorganizacij "reformirovali" zemel'nuju reform (Land reform in Russia in 1990-2000-ies, or how land reform was "reformed" during the departmental reorganization). Jurnal Institucional'nyh Isledovanij (Journal of Institutional Studies), vol. ${ }^{\circ}$, $\mathrm{n}^{\circ}$ 2, pp. 84-98.

Dufy C. (2015). Redefining Business Values in Russia : the Boundaries of Globalization and Patriotism in Contemporary Russian Industry. Europe-Asia Studies, vol. 67, n 1, pp. 84-101.

Dufy C., Barsukova S. (2017). Sécurité alimentaire et marché. Représentations des acteurs du monde agricole dans la Russie des années 1990-2010. Revue d'études comparatives Est-Ouest, vol. 48, n 1-2, pp. 57-84.

Götze L., Koester U., Glauben T., Bulavin R. (2015). The Rouble Crisis and Russian 
Wheat Export Controls. Intereconomics, vol. 50, n० 4, pp. 227-233.

Grouiez P. (2011). Les stratégies des exploitations agricoles et la multifonctionnalité de l'agriculture russe. Revue d'études comparatives Est-Ouest, vol. 42, n² 2, pp. 139-164.

Grouiez P. (2012a). Des kolkhozes à l'agrobusiness en Russie. Études rurales, n 190.

Grouiez P. (2012b). OMC : quel impact pour le secteur agricole russe? Russie. Nei. Reports, $\mathrm{n}^{\circ} 15$, décembre, pp. 1-33.

Hufbauer G. C., Schott J. J., Elliott A. K. (2007). Economic Sanctions Reconsidered. Peterson Institute for International Economics.

Kirtchik O. (2009). Les défis de la politique agricole en Russie dans le contexte de la globalisation libérale. Mondes en mouvement, dossier.

Lezean E. (2011), L'agriculture russe en transition : entre stagnation et modernisation post-soviétiques. Économie rurale, $\mathrm{n}^{\circ} 325$ 326, pp. 159-173.

Lindsay J. M. (1986). Trade Sanctions as Policy Instruments: A Re-examination. International Studies Quarterly, $\mathrm{n}^{\circ}$ 30, pp. 153-173.

List F. (1857). Système national d'économie politique. Trad. de l'allemand par Heni Richelot. Paris, Capelle, $2^{\mathrm{e}}$ éd.

Martin P., Mayer T., Thöenig M. (2008). Make Trade not War. Review of Economic Studies, $\mathrm{n}^{\circ} 75$, pp. 865-900.
Matelly S., Gomez C., Carcanague S. (2017). Performance des sanctions internationales, Typologie : étude de cas. Rapport final PERSAN juin 2017, IRIS, CSFRS.

Néfédova T., Eckert D. (2003). L'agriculture russe après 10 ans de réformes : transformation et diversité. L'Espace géographique, vol. $32, n^{\circ} 4$, pp. 289-300.

Porter R. C. (1979). International Trade and Investment Sanctions Potential Impact on the South Africa. Journal of Conflict Resolution, vol. 23, n 4 , pp. 579-612.

Rosreestr (2012). Gosudarstvennyj (nacional'nyj) doklad o sostojanii i ispol'zovanii zemel' v Rossijskoj Federacii v 2011 godu (The state [national] report is about the status and use of lands in the Russian Federation in 2011). Moscow. Federal'naja sluzhba gosudarstvennoj registracii, kadastra i kartografii (Rosreestr) (Federal Service for State Registration, Cadastre and Cartography), $248 \mathrm{p}$.

Rylko D., D. Khotko, A. Svetlana, N. Yunosheva, I. Glazunova (2015). Country Report: Russian Federation. Agricis Trade.

Sapir J. (2008). Le nouveau XXI' siècle. Du siècle américain au retour des nations. Paris, Seuil.

Yefimov V. (2001). Continuité et recomposition des régimes agraires russes dans le siècle. Économie et Société, vol. 39, n 9-10, pp. 1439-1473. 